ctp-scu/2014003

\title{
New Analytic Solutions in String Field Theory: Towards Collective Higher Spin Vacuum
}

\author{
Dimitri Polyakov ${ }^{\dagger}$ \\ Center for Theoretical Physics (1) \\ College of Physical Science and Technology \\ Sichuan University, Chengdu 610064, China \\ Center for Quantum Space-Time $(C Q U e S T)^{(2)}$ \\ Sogang University \\ Seoul 121-742, Korea \\ Institute for Information Transmission Problems $(\text { IITP) })^{(3)}$ \\ Bolshoi Karetny per. 19/1 \\ 127994 Moscow, Russia
}

\begin{abstract}
We construct analytic solutions in cubic open superstring field theory at higher superconformal ghost numbers. The solutions are the pure ghost ones, given by combinations of Bell polynomials of bosonized superconformal ghost fields multiplied by exponents of the bosonized ghosts. Based on the structure of the solutions, we conjecture them to describe the ghost part of collective vacuum for higher spin modes in open string theory.
\end{abstract}

August 2014

$\dagger$ polyakov@sogang.ac.kr ; twistorstring@gmail.com 


\section{Introduction}

String theory is known to be a powerful tool to approach problems of describing consistently interacting higher spin (HS) field theories, as well as higher spin holography. In string theory, the higher spin modes appear naturally as vertex operators and the symmetries of the higher spin algebra are realized in terms of the operator algebras of these vertices [1], [2], [3], [1], [4], [5], [6]. As the on-shell constraints and the symmetry transformations on higher spin fields in space-time follow from the BRST conditions on the corresponding vertex operators in open or closed string theory, the $N$-point correlation functions of higher spin vertex operators also define the gauge-invariant HS interactions and, in the AdS case, the holographic couplings in dual CFT [7] Unfortunately, however, the on-shell string theory is background-dependent and it is generally hard to approach string theory in AdS space beyond semiclassical limit. On the other hand, open string field theory (OSFT) is currently our best hope to advance towards background independent formulation of strings, with the OSFT equations of motion in the cubic-like theory formally reminiscent of the relations for the master fields in the Vasiliev's equations in the unfolding formalism for higher spins - with the star products naturally appearing in the both theories [8], [9], [10], [11], [12], [2], [13], [14], [15], [16], [17], 118], [19], [20], [21] At the same time, the form of the vertex operators in RNS string theory, describing the higher spin gauge fields in the frame-like formalism [22] already carries a strong hint on their relevance to background independence and emergent $A d S$ geometry. Namely, consider open string vertex operators for Vasiliev type two-row higher spin gauge fields $\Omega_{m}^{a_{1} \ldots a_{s-1} \mid b_{1} \ldots b_{t}}(x) \equiv$ $\Omega^{s-1 \mid t}(x)(0 \leq t \leq s-1)$ [3], [23], [2], [24] where $m$ is the curved $d$-dimensional space index and $a, b$ indices (corresponding to rows of lengths $s-1$ and $t$ ) label $d$-dimensional tangent space. In case of $t=s-3$ the expression for the spin $s$ operator particularly simplifies and is given by:

$$
\begin{array}{r}
V_{s-1 \mid s-3}(p) \equiv \Omega_{m}^{a_{1} \ldots a_{s-1} \mid b_{1} \ldots b_{s-3}}(p) V_{a_{1} \ldots a_{s-1} \mid b_{1} \ldots b_{s-3}}^{m}(p) \\
=\Omega_{m}^{a_{1} \ldots a_{s-1} \mid b_{1} \ldots b_{s-3}}(p) \oint d z e^{-s \phi} \psi^{m} \partial \psi_{b_{1}} \partial^{2} \psi_{b_{2} \ldots} \ldots \partial^{s-3} \psi_{b_{s-3}} \partial X_{a_{1} \ldots \partial X_{a_{s-2}} e^{i p X}}
\end{array}
$$

at minimal negative picture $-s$. The manifest expressions for the spin $s$ operators with $0 \leq t<s-3$ are generally more complicated, however, at their canonical pictures equal to $-2 s+t+3$, they can be related to the operator $V_{a_{1} \ldots a_{s-1} \mid b_{1} \ldots b_{s-3}}^{m}$ (1) by

$$
: \Gamma^{s-t-3} \Omega_{m}^{a_{1} \ldots a_{s-1} \mid b_{1} \ldots b_{t}}(p) V_{a_{1} \ldots a_{s-1} \mid b_{1} \ldots b_{t}}^{m}:(p)=\Omega_{m}^{a_{1} \ldots a_{s-1} \mid b_{1} \ldots b_{s-3}}(p) V_{a_{1} \ldots a_{s-1} \mid b_{1} \ldots b_{s-3}}^{m}(p)
$$


where $\Gamma=: e^{\phi} G$ : is the picture-changing operator satisfying : $\Gamma^{m} \Gamma^{n}:=: \Gamma^{m+n}$ : $+\left\{Q_{b r s t}, \ldots\right\}, G$ is the full matter + ghost worlsheet supercurrent and

: $\Gamma^{n}: \sim: e^{n \phi} G \partial G \ldots \partial^{n-1} G$ : The operator identity (2) particularly entails a set of generalized torsion zero constraints relating the space-time extra fields in the frame-like formalism for the higher spins [22]:

$$
\Omega s-1 \mid s-3(x) \sim \partial^{s-3-t} \Omega^{s-1 \mid s-3}(x)
$$

Although the canonical pictures for the $V^{s-1 \mid t}$ (defined by the singularity order in the asymtotic behavior of the supermoduli approaching the insertion point of a vertex operator) are different for various $t$ values, this shouldn't be confused with ghost cohomology ranks which are the same for all the vertex operators of the extra fields with given $s$ and only depend on the spin value $s$; that is, all the operators for the frame-like fields of spin $s \geq 3$ are the elements of $H_{-s} \sim H_{s-2}$ [6], [22]. In the leading order, the low-energy equations of motion for $\Omega^{s-1 \mid t}$ extra fields are defined by the Weyl invariance constraints on their vertex operators. Naively, since the operators (1) are massless and originally are defined in flat space-time, one would expect the low-energy equations of motions to be given simply by $\beta_{\Omega} \sim p^{2} \Omega^{s-1 \mid t}(p)=0$ in the leading order. However, as was explained in [22, due to nontrivial ghost dependence of the operators in $H_{-s} \sim H_{s-2}$ there are anomalous "masslike" terms appearing in the low-energy equations of motion which, in the leading order, are actually given by

$$
\begin{gathered}
\beta_{m}^{a_{1} \ldots a_{s-1}}=-p^{2} \Omega_{m}^{a_{1} \ldots a_{s-1} \mid b_{1} \ldots b_{t}}(p)+\Sigma_{1}\left(a_{1} \mid a_{2}, \ldots a_{s-1}\right) p_{d} p^{a_{1}} \Omega_{m}^{a_{2} \ldots a_{s-1} d \mid b_{1} \ldots b_{t}} \\
-\frac{1}{2} \Sigma_{2}\left(a_{s-2}, a_{s-1} \mid a_{1}, \ldots, a_{s-3}\right) p^{a_{s-1}} p^{a_{s-2}}\left(\Omega_{m}^{\prime}\right)^{a_{1} \ldots a_{s-3} \mid b_{1} \ldots b_{t}}-4(s-1) \Omega_{m}^{a_{1} \ldots a_{s-1} \mid b_{1} \ldots b_{t}}
\end{gathered}
$$

where $\Sigma_{1,2}$ are the Fronsdal's symmetrization operators 25 and the prime stands for taking a trace over a couple of indices. For $t=0$, these leading order equations of motion coincide with those for the Fronsdal's massless higher spin fields in $A d S$ background, polarized along the boundary of $A d S$, with the "mass-like" term related to the covariant derivative of the Laplacian in $A d S$. This leading order calculation already provides an example how curved geometrical backgrounds (in this case, $A d S$ ) typically appear in calculations involving vertex operators with nontrivial ghost dependence. Unfortunately, the calculation performed in [22] is hard to generalize beyond the leading order, particularly because the operators with different $t$ values mix with each other at the level of 3-point functions and beyond, so the straightforward $\beta$-function calculations become cumbersome 
and practically insurmountable. Given the fact that the conformal $\beta$-function is essentially an off-shell object and, in case of the higher spin operators, demonstrates the background change, this clearly makes a string field theory, extended to higher superconformal ghost numbers, a natural framework to approach the problem of higher spin interactions from string-theoretic point of view. First of all, recall that the background independence in cubic string field theory implies that the equations of motion:

$$
Q \Psi+\Psi \star \Psi=0
$$

are invariant under the shift $\Psi \rightarrow \Psi+\Psi_{0}$ where $\Psi_{0}$ is a solution of (5), provided that the BRST charge is shifted according to: $Q \rightarrow Q+\Psi_{0}$, implying $Q \Psi \rightarrow \tilde{Q} \Psi=Q \Psi+$ $\Psi_{0} \star \Psi+\Psi \star \Psi_{0}$ for any string field $\Psi$. It is important that, in this approach, the new BRST charge, $\tilde{Q}$ defines new BRST cohomology and string field theory around certain new geometrical background, even though the original theory is often defined around the flat vacuum (making it possible to compute the off-shell correlators). For this reason, exact analytic solutions in OSFT are crucial ingredients to analyze the off-shell interactions in various space-time geometries (such as AdS), holding a key to background independence. Unfortunately, because of the complexity of the equations (5) very few concrete examples of the analytic OSFT solutions are known so far. One remarkable example is of course the nonperturbative tachyon vacuum solution proposed by Schnabl [26], generalized in many subsequent importants papers [27], [28], [29] and particularly used to prove the Sen's conjectures on tachyon condensation [26], [27] The solutions found by Schnabl [26] particularly used the basis of the wedge states where the star product simplifies significantly [16], [26] Nevertheless, because of the complexity of the star product (7) calculating the star products of string fields is generally an extremely cumbersome and tedious problem. The reason for this complexity is that, in terms of correlation functions in OSFT, the star product involves conformal transformations mapping the worldsheets of interacting strings to wedges of a single disc. For example, in case of $N$ interacting strings the transformation mapping the worldsheet of the $\mathrm{n}$-th string to the $\mathrm{n}$-th wedge $(1 \leq n \leq N)$ is given by

$$
f_{n}^{N}(z)=e^{\frac{i \pi(n-1)}{N}}\left(\frac{1-i z}{1+i z}\right)^{\frac{2}{N}}
$$

, and the star product of two string fields is defined according to

$$
<<\Phi ; \Psi_{1} \star \Psi_{2}>>=<f_{1}^{3} \circ \Phi(0) f_{2}^{3} \circ \Psi_{1}(0) f_{3}^{3} \circ \Psi_{2}(0)>
$$


for an arbitrary string field $\Phi$ Here $f \circ \Psi$ is conformal transformation acting on all the operators (generically, off-shell) entering $\Psi$. For primary fields $V_{h}$ of conformal dimension $h$, the transformation is simply given by

$$
f \circ V_{h}(0)=\left(\frac{d f}{d z}\right)^{h} V_{h}(f(0))
$$

and in case if descendents are given by the derivatives of the primaries the transformation is given by differentiating (8). However, for generic non-primaries the global transformation laws are far more complicated and cannot be reduced to differentiating the combinations of (8) (e.g. recall the simplest example - the global transformation law of a stress tensor involving Schwarzian derivatives) Therefore a generic string field transforms under (6) in a complicated manner that is hard to control, so straightforward calculation of the star product through correlators is not accessible. However, as we point out in this work, there exists a substantially large class of operators for which the global conformal transformations simplify significantly, having an elegant and compact form. This class includes Bell polynomials of bosonized superconformal ghost fields multiplied by exponents of the bosonized ghosts. These operators altogether form an invariant subspace under global conformal transformations, making it possible to directly deduce the star product from the correlation functions for the string fields restricted to this subspace. In this paper we consider the cubic superstring field theory [10], [30, [12] We impose no restrictions on superconformal ghost numbers of string field components, allowing them to have any positive or negative pictures. We furthermore impose weak cohomology condition (44) on the string field replacing the standard gauge fixing (see below); this condition ensures that string field components at different superconformal ghost numbers are not mixed by picture changing For this reason, there is no need for the midpoint insertions of inverse picture-changing operators (unlike the cases when the ghost number and picture of string fields are fixed or the model of democratic superstring field theory considered in [31]) In this paper we particularly propose an ansatz for an analytic solution in string field theory with the structure:

$$
\begin{array}{r}
\Psi=\Psi^{(+)}+\Psi^{(-)} \\
\Psi^{(+)}=\sum_{N=1}^{\infty} \sum_{n=0}^{N-2} \lambda_{N}^{n} c e^{\chi+N \phi} B_{n}^{\left[\alpha_{n}, \beta_{n}, \gamma_{n}\right]}(\phi, \chi, \sigma) \\
\Psi^{(-)}=\sum_{N=1}^{\infty} \sum_{n=0}^{N-2} \lambda_{N}^{n} c e^{-(N+2) \phi} B_{n}^{\left[\alpha_{n}, \beta_{n}, \gamma_{n}\right]}(\phi, \chi, \sigma)
\end{array}
$$


The limits of summations over $n$ are related to the ghost cohomology constraints on $\Psi^{(+)}$and $\Psi^{(-)}$(see below). The $\lambda_{N^{-}}^{n}$ coefficients in front of $c e^{\chi+N \phi}$ and $c e^{-(N+2) \phi}$ are chosen equal in order to preserve the isomorphism between negative and positive ghost cohomologies $H_{n} \sim H_{-(n+2)}$ [6] and $\alpha_{n}, \beta_{n}, \gamma_{n}$ are certain numbers chosen to satisfy ghost cohomology constraints on $\Psi$ (see below). The Bell polynomials in bosonized ghost fields can be computed according to

$$
B_{n}^{\left[\alpha_{n}, \beta_{n}, \gamma_{n}\right]}(\phi, \chi, \sigma)=e^{-\alpha_{n} \phi-\beta_{n} \chi-\gamma_{n} \sigma}(z) \frac{d^{n}}{d z^{n}} e^{\alpha_{n} \phi+\beta_{n} \chi+\gamma_{n} \sigma}(z)
$$

implying $x_{k}=\alpha_{k} \partial \phi+\beta_{k} \partial \chi+\gamma_{k} \partial \sigma$ in the formal definition (14), (15). The analytic solution with the structure (9) is then calculated in our work in terms of recurrence relations satisfied by $\lambda_{N}^{n}$ coefficients:

$$
\lambda_{N}^{n}=\sum_{N_{1}, N_{2}=1}^{N_{1}+N_{2}=N-2} \sum_{n_{1}=0}^{N_{1}-1} \sum_{n_{2}=0}^{N_{2}-1} \rho_{N \mid N_{1} ; N_{2}}^{n \mid n_{1} ; n_{2}} \lambda_{N_{1}}^{n_{1}} \lambda_{N_{2}}^{n_{2}}
$$

Our purpose is to determine the coefficients: $\rho_{N \mid N_{1}, N_{2}}^{n \mid n_{1} ; n_{2}}$ by directly computing the star product, i.e. the relevant correlators

$$
<<\Psi, Q \Psi>>=<\Psi(0) I \circ Q \Psi(0)>
$$

and

$$
<<\Psi, \Psi \star \Psi>>=<h \circ f_{1}^{3} \circ \Psi(0) h \circ f_{2}^{3} \circ \Psi(0) h \circ f_{3}^{3} \circ \Psi(0)>
$$

where $I(z)=-\frac{1}{z}$ and

$$
f_{k}^{n}(z)=e^{\frac{i \pi(k-1)}{n}}\left(\frac{1-i z}{1+i z}\right)^{\frac{2}{n}}
$$

maps the worldsheets of $n$ interacting strings putting them together on a single disc and

$$
h(z)=-i \frac{z-1}{z+1}
$$

maps this disc back to the halfplane.

The rest of this paper is organized as follows. In section 2 we review basic facts about Bell polynomials and derive the global conformal transformation rules for operators entering the proposed ansatz for the exact analytic solution. In the the Section 3 we compute the relevant correlators in OSFT and derive the recursion relations for the coefficients defining the solution. In the concluding section we discuss the relevance of the solution to higher spin algebras in $A d S$ and possible generalizations. 


\section{Bell Polynomials and Global Conformal Transformations}

The standard definition of the complete Bell polynomials [32], [33], [34] $B_{n}\left(x_{1}, \ldots x_{n}\right)$ is given by

$$
B_{n}\left(x_{1}, \ldots x_{n}\right)=\sum_{k=1}^{n} B_{n \mid k}\left(x_{1}, \ldots x_{n-k+1}\right)
$$

where $B_{n \mid k}\left(x_{1}, \ldots x_{n-k+1}\right)$ are the partial Bell polynomials defined according to

$$
B_{n \mid k}\left(x_{1}, \ldots x_{n-k+1}\right)=\sum_{p_{1}, \ldots p_{n-k+1}} \frac{n !}{p_{1} ! \ldots p_{n-k+1} !} x_{1}^{p_{1}}\left(\frac{x_{2}}{2 !}\right)^{p_{2}} \ldots\left(\frac{x_{n-k+1}}{(n-k+1) !}\right)^{p_{n-k+1}}
$$

with the sum taking over all the combinations of non-negative $p_{j}$ satisfying

$$
\begin{gathered}
\sum_{j=1}^{n-k+1} p_{j}=k \\
\sum_{j=1}^{n-k+1} j p_{j}=n
\end{gathered}
$$

In number theory, Bell polynomials are known to satisfy a number of useful and beautiful identities and properties, with some of them important for the calculations performed in this paper [32], [33] Just to mention a couple of examples,

$$
B_{n \mid k}(1, \ldots 1)=S(n, k)
$$

is the second kind Stirling number and $B_{n \mid k}(0 !, 1 !, \ldots,(n-k) !)$ can be expressed in terms of combinations of Bernoulli numbers (note the appearance of Bernoulli numbers in the analytic SFT solutions describing the tachyonic vacuum [26]). Also, given a Taylor's expansion of a function $f(x)=\sum_{n} \frac{a_{n} x^{n}}{n !}$ one has $e^{f}(x)=\sum_{n} B_{n}\left(a_{1}, . . a_{n}\right) \frac{x^{n}}{n !}$ in terms of formal series, so e.g. vertex operators in string theory are typically given by combinations of Bell polynomials in the expansion modes. Note that the SFT ansatz (9) is actually bilinear in Bell polynomials of the ghost expansion modes. If one identifies $x_{n}=\partial^{n} \phi(z)$, where $\phi(z)$ is some scalar field, one obtains Bell polynomials in derivatives of $\phi$; note that in the particular case $\phi(z) \sim z^{2}$ this would reduce to Hermite polynomials in $z$. Other useful objects to define are the Bell generators

$$
H_{n}\left(y \mid x_{1}, \ldots, x_{n}\right)=\sum_{k=1}^{n} B_{n \mid k}\left(x_{1}, \ldots, x_{n-k+1}\right) y^{n}
$$


and more generally

$$
G_{n}\left(y_{1} \ldots y_{n} \mid x_{1}, \ldots, x_{n}\right)=\sum_{k=1}^{n} B_{n \mid k}\left(x_{1}, \ldots, x_{n-k+1}\right) y_{1}, \ldots, y_{k}
$$

In the context of two-dimensional CFT, one can think of Bell polynomials as higher derivative generalizations of the Schwarzian derivative, appearing in the global conformal transformation law for the stress tensor. That is, under $z \rightarrow f(z)$ one has

$$
T(z) \rightarrow\left(\frac{d f}{d z}\right)^{2} T(f(z))+\frac{c}{12} S(f(z))
$$

where the Schwarzian derivative:

$$
S(f(z))=\left(\frac{f^{\prime \prime}(z)}{f^{\prime}(z)}\right)^{\prime}-\frac{1}{2}\left(\frac{f^{\prime \prime}(z)}{f^{\prime}(z)}\right)^{2}
$$

can be expressed in terms of the second order Bell polynomials in the log of $f^{\prime}$, with $x_{k} \equiv \frac{d^{k-1}}{d z^{k-1}} \log \left(f^{\prime}\right)$ :

$$
\begin{aligned}
S(f(z)) & =B_{2 \mid 1}\left(\log \left(f^{\prime}\right), \frac{d \log \left(f^{\prime}\right)}{d z}\right)-\frac{1}{2} B_{2 \mid 2}\left(\log \left(f^{\prime}\right)\right) \\
& \equiv-2 H_{2}\left(-\frac{1}{2} \mid \log \left(f^{\prime}\right)\right) \equiv-2 B_{2}\left(-\frac{1}{2} \log \left(f^{\prime}\right)\right)
\end{aligned}
$$

where, for the sake of brevity, we adopt the notation:

$$
B_{n}(g(x)) \equiv B_{n}\left(\partial g, \ldots \partial^{n} g\right)=\left.B_{n}\left(x_{1}, \ldots x_{n}\right)\right|_{x_{k}=\partial_{x}^{k} g(x) ; k=1, \ldots, n}
$$

for any function $g(x)$. This point is of importance as the higher order Bell polynomials will naturally enter the global transformation law for the string fields of the type (9) (see below). We now turn to the question of finding global conformal transformation law for the OSFT ansatz (9). Our strategy will be to find the infinitezimal form of the transformation first and then to deduce the global transformation by requiring it to reproduce the infinitezimal one while preserving its form under the composition of two global transformations. We start with the infinitezimal transformation following from the OPE of the stress tensor $T(z)$ with $B_{n}^{\left[\alpha_{n}, \beta_{n}, \gamma_{n}\right]} e^{[\alpha \cdot \beta \gamma]}$ where $\alpha, \alpha_{n}, \ldots$ are some numbers and

$$
e^{[\alpha \cdot \beta \gamma]} \equiv e^{\alpha \phi+\beta \chi+\gamma \sigma}
$$

in our notations. Consider the transformation of $B_{n}^{\left[\alpha_{n}, \beta_{n}, \gamma_{n}\right]}$ first. It is calculated easily noting that

$$
B_{n}^{\left[\alpha_{n}, \beta_{n}, \gamma_{n}\right]} e^{\left[\alpha_{n} \cdot \beta_{n} \gamma_{n}\right]}=\partial^{n} e^{\left[\alpha_{n} \cdot \beta_{n} \gamma_{n}\right]}
$$


and

$$
T(z) e^{\left[\alpha_{n} \cdot \beta_{n} \gamma_{n}\right]}(w)=\frac{h^{\left[\alpha_{n}, \beta_{n}, \gamma_{n}\right]}}{(z-w)^{2}}+\frac{\partial e^{\left[\alpha_{n} \cdot \beta_{n} \gamma_{n}\right]}}{z-w}+\ldots
$$

where

$$
h^{\left[\alpha_{n}, \beta_{n}, \gamma_{n}\right]}=\frac{1}{2}\left(-\alpha^{2}+\beta^{2}+\gamma^{2}\right)-\alpha-\frac{\beta}{2}-\frac{3 \gamma}{2}
$$

stand for conformal dimensions of the exponents (with similar notations below). One then easily computes:

$$
\begin{array}{r}
T(z) B_{n}^{\left[\alpha_{n}, \beta_{n}, \gamma_{n}\right]} e^{\left[\alpha_{n}, \beta_{n} \gamma_{n}\right]}(w) \\
=\sum_{k=0}^{n+1} \frac{n !}{(n-k+1) !} \frac{k h^{\left[\alpha_{n}, \beta_{n}, \gamma_{n}\right]}+n-k+1}{(z-w)^{k+1}} B_{n-k+1}^{\left[\alpha_{n}, \beta_{n}, \gamma_{n}\right]} e^{\left[\alpha_{n}, \beta_{n} \gamma_{n}\right]}(w) \\
+\ldots
\end{array}
$$

To compute the variation of $B_{n}^{\left[\alpha_{n}, \beta_{n}, \gamma_{n}\right]}$ under infinitezimal conformal transformation, we note that

$$
\begin{array}{r}
\delta_{\epsilon}\left(B_{n}^{\left[\alpha_{n}, \beta_{n}, \gamma_{n}\right]}\right) e^{\left[\alpha_{n} \cdot \beta_{n} \gamma_{n}\right]} \\
=\delta_{\epsilon}\left(B_{n}^{\left[\alpha_{n}, \beta_{n}, \gamma_{n}\right]} e^{\left[\alpha_{n} \cdot \beta_{n} \gamma_{n}\right]}\right)-B_{n}^{\left[\alpha_{n}, \beta_{n}, \gamma_{n}\right]} \delta_{\epsilon}\left(e^{\left[\alpha_{n} \cdot \beta_{n} \gamma_{n}\right]}\right) \\
\text {-overlap }
\end{array}
$$

with the overlap contribution stemming from the leading order singularity in the OPE of the quadratic part of the stress energy tensor with one of $\partial \phi, \partial \chi, \partial \sigma$ coupling with $B_{n}$ and another with the exponent.

To compute this contribution, one first has to calculate the OPE of $\partial \phi$ with $B_{n}^{\left[\alpha_{n}, \beta_{n}, \gamma_{n}\right]}$ (analogously, for $\partial \chi$ and $\partial \sigma$ ) This can be done directly, by using straightforward expression for $B_{n}$ :

$$
B_{n}^{\left[\alpha_{n}, \beta_{n}, \gamma_{n}\right]}=\sum_{m=1}^{n} \sum_{n \mid p_{1} \ldots p_{m}} \frac{n !}{p_{1} ! \ldots p_{m} ! q_{p_{1}} ! \ldots q_{p_{m}} !} \prod_{j=1}^{m}\left(\alpha \partial^{p_{j}} \phi+\beta \partial^{p_{j}} \chi+\gamma \partial^{p_{j}} \sigma\right)
$$

where $n \mid p_{1} \ldots p_{m}$ are the ordered partitions of $n$ into $\mathrm{m}$ numbers $0<p_{1} \leq p_{2} \ldots \leq p_{m}$ and $q_{p_{j}}$ are a multiplicities of $p_{j}$ 's entering a partition. Then the OPE is easily computed to give

$$
\partial \phi(z) B_{n}^{\left[\alpha_{n}, \beta_{n}, \gamma_{n}\right]}(w)=-\alpha_{n} \sum_{k=1}^{n} \frac{n !}{(n-k) !} \frac{B_{n-k}^{\left[\alpha_{n}, \beta_{n}, \gamma_{n}\right]}}{(z-w)^{k+1}}+O(z-w)^{0}
$$


and similarly for $\partial \chi$ and $\partial \sigma$. Using (30), the overlap contribution to (28) is given by

$$
T(z) B_{n}^{\left[\alpha_{n}, \beta_{n}, \gamma_{n}\right]}(w)=\left(-\alpha^{2}+\beta^{2}+\gamma^{2}\right) \sum_{k=1}^{n} \frac{B_{n-k}^{\left[\alpha_{n}, \beta_{n}, \gamma_{n}\right]}}{(z-w)^{k+2}}
$$

Combining (28) and (31), the infinitezimal conformal transformation of $B_{n}^{\left[\alpha_{n}, \beta_{n}, \gamma_{n}\right]}$ is given by

$$
\begin{array}{r}
\delta_{\epsilon} B_{n}^{\left[\alpha_{n}, \beta_{n}, \gamma_{n}\right]}=\epsilon \partial B_{n}^{\left[\alpha_{n}, \beta_{n}, \gamma_{n}\right]}+n \partial \epsilon B_{n}^{\left[\alpha_{n}, \beta_{n}, \gamma_{n}\right]} \\
+\sum_{k=2}^{n+1} \frac{n !}{(n-k+1) ! k !} \partial^{k} \epsilon(z) k h^{\left[\alpha_{n}, \beta_{n}, \gamma_{n}\right]}+n-k+1+\left(\alpha_{n}^{2}-\beta_{n}^{2}-\gamma_{n}^{2}\right) B_{n-k+1}^{\left[\alpha_{n}, \beta_{n}, \gamma_{n}\right]}(z)
\end{array}
$$

Finally, the infinitezimal conformal transformation of terms entering the OSFT ansatz (9) is given by:

$$
\begin{array}{r}
\delta_{\epsilon}\left(B_{n}^{\left[\alpha_{n}, \beta_{n}, \gamma_{n}\right]} e^{[\alpha, \beta, \gamma]}\right) \delta_{\epsilon}\left(B_{n}^{\left[\alpha_{n}, \beta_{n}, \gamma_{n}\right]}\right) e^{[\alpha, \beta, \gamma]}+B_{n}^{\left[\alpha_{n}, \beta_{n}, \gamma_{n}\right]} \delta_{\epsilon}\left(e^{[\alpha, \beta, \gamma]}\right) \\
+ \text { overlap }
\end{array}
$$

with the overlap contribution in (33) stemming from the leading order singularity in the OPE of the quadratic part of $T(z)$ with $B_{n}^{\left[\alpha_{n}, \beta_{n}, \gamma_{n}\right]} e^{[\alpha, \beta, \gamma]}$ with one of $\partial(\phi, \chi, \sigma)$ contracting with $B_{n}$ and another with $e^{[\alpha, \beta, \gamma]}$ so the overall infinitezimal transformation of the string field components is

$$
\begin{aligned}
\delta_{\epsilon}\left(B_{n}^{\left[\alpha_{n}, \beta_{n}, \gamma_{n}\right]} e^{[\alpha, \beta, \gamma]}\right)= & \epsilon \partial\left(B_{n}^{\left[\alpha_{n}, \beta_{n}, \gamma_{n}\right]} e^{[\alpha, \beta, \gamma]}\right)+\partial \epsilon\left(n+h^{[\alpha, \beta, \gamma]}\right) B_{n}^{\left[\alpha_{n}, \beta_{n}, \gamma_{n}\right]} e^{[\alpha, \beta, \gamma]} \\
& +\sum_{k=2}^{n+1} \frac{n !}{(n-k+1) ! k !} \partial^{k} \epsilon(z)\left[k h^{\left[\alpha_{n}, \beta_{n}, \gamma_{n}\right]}+n-k+1\right. \\
& \left.+\left(\alpha_{n}^{2}-\alpha_{n} \alpha-\beta_{n}^{2}+\beta_{n} \beta-\gamma_{n}^{2}+\gamma_{n} \gamma\right)\right] B_{n-k+1}^{\left[\alpha_{n}, \beta_{n}, \gamma_{n}\right]} e^{[\alpha, \beta, \gamma]}(z)
\end{aligned}
$$

Given the infinitezimal transformation (34) with some effort one can deduce the correct form of the global conformal transformation of the string field components (9) under $z \rightarrow$ $f(z)$ by requiring that:

1) it reproduces the transformation (34) for $f(z)=z+\epsilon(z)$

2) its form is preserved under the composition of two transformations

(as in the standard derivation of the transformation law for the stress tensor, leading to the appearance of Schwarzian derivative, which is simply the second order Bell polynomial in the $\log$ of $f^{\prime}(22)$.) 
Regarding the first condition, note that

$$
\left.B_{n}\left(\log \left(f^{\prime}(z)\right)\right)\right|_{f(z)=z+\epsilon(z)}=\partial^{n} \epsilon(z)+O\left(\epsilon^{2}\right)
$$

Regarding the second, note the binomial property of $B_{n}(f)$ :

$$
B_{n}(f(x)+g(x)) \equiv B_{n}\left(\partial(f+g), \ldots, \partial^{n}(f+g)\right)=\sum_{k=0}^{n} \frac{n !}{k !(n-k) !} B_{k}(f) B_{n-k}(g)
$$

which obviously follows from the chain rule applied to the derivative $\partial_{z}^{n} e^{f(z)+g(z)}$ and, finally, $\frac{d}{d z} \log (g(f(z)))=\log \left(g^{\prime}(f)\right)+\log \left(f^{\prime}(z)\right)$ for the composition of two conformal transformations $f$ and $g$. This altogether fixes the form of the global conformal transformation according to

$$
\begin{array}{r}
B_{n}^{\left[\alpha_{n}, \beta_{n}, \gamma_{n},\right]} e^{[\alpha, \beta, \gamma]}(z) \rightarrow\left(\frac{d f}{d z}\right)^{n+h^{[\alpha, \beta, \gamma]}} B_{n}^{\left[\alpha_{n}, \beta_{n}, \gamma_{n}\right]} e^{[\alpha, \beta, \gamma]}(f(z)) B_{n-k+1}^{\left[\alpha_{n}, \beta_{n}, \gamma_{n}\right]} e^{[\alpha, \beta, \gamma]}(f(z)) \\
+\sum_{k=2}^{n+1} \frac{n !}{k !(n-k+1) !}\left(\frac{d f}{d z}\right)^{n-k+1+h^{[\alpha, \beta, \gamma]}} B_{k-1}\left(\lambda\left(k, n, h^{\left[\alpha_{n}, \beta_{n}, \gamma_{n}\right]}\right) \log \left(f^{\prime}(z)\right)\right) \\
\times B_{n-k+1}^{\left[\alpha_{n}, \beta_{n}, \gamma_{n}\right]} e^{[\alpha, \beta, \gamma]}(f(z))
\end{array}
$$

with the weight factor $\lambda$ given by

$$
\begin{array}{r}
\lambda\left(k, n, h^{\left[\alpha_{n}, \beta_{n}, \gamma_{n}\right]}\right)=k h^{\left[\alpha_{n}, \beta_{n}, \gamma_{n}\right]}+n-k+1 \\
+\alpha_{n}^{2}-\alpha_{n} \alpha-\beta_{n}^{2}+\beta_{n} \beta-\gamma_{n}^{2}+\gamma_{n} \gamma
\end{array}
$$

This defines the global conformal transformations for all the string field components (9) under $z \rightarrow f(z)$. Finally, to prepare for the computation of the SFT correlators, we need to determine the BRST transformation of the string field (9), in order to compute $<<\Psi, Q \Psi>>=<\Psi I \circ(Q \Psi)>$ where $I$ is the conformal transformation $z \rightarrow w=-\frac{1}{z}$. Since all the components of $\Psi$ carry $b-c$ ghost number +1 , it shall be sufficient to compute the terms of $Q \Psi$ carrying the $b-c$ ghost number +2 , that is, the commutator of $\Psi$ with the stress tensor part of $Q$ given by $\oint \frac{d z}{2 i \pi}(c T-b c \partial c)$. Moreover, since $\Psi$ is pure ghost, it is suffucient to consider the ghost part of $T(z)$. Another simplification stems from the fact that the bosonized expression for $c T_{b-c}-b c \partial c$ (where $T_{b-c}=\frac{1}{2}(\partial \sigma)^{2}+\frac{3}{2} \partial^{2} \sigma$ is the $b-c$ part of the stress-tensor) given by

$$
: c T_{b-c}-b c \partial c:=e^{\sigma}\left(T_{b-c}(z)-\partial^{2} \sigma\right)
$$


so the effect of the second term is just reducing the background charge by 1 unit; in particular, in our computations of $I \circ(Q \Psi)=Q(I \circ \Psi)$ this results in effective shifts of the conformal dimensions of $e^{\left[\alpha_{n}, \beta_{n}, \gamma_{n}\right]}$ according to

$$
h^{\left[\alpha_{n}, \beta_{n}, \gamma_{n}\right]} \rightarrow \tilde{h}^{\left[\alpha_{n}, \beta_{n}, \gamma_{n}\right]}=h^{\left[\alpha_{n}, \beta_{n}, \gamma_{n}\right]}+\gamma_{n}
$$

Straightforward computation of $Q(I \circ \Psi)$ then gives:

$$
\begin{array}{r}
\left.Q\left(I \circ B_{n}^{[\alpha, \beta, \gamma,]} e^{[\alpha, \beta, \gamma]}\right)(w)\right|_{w=-\frac{1}{z}} \\
=\sum_{k=1}^{n+1} \frac{n !}{k !} \times\left[\left(k-\delta_{1}^{k}\right) h^{\left[\alpha_{n}, \beta_{n}, \gamma_{n}\right]}+\delta_{1}^{k} h^{[\alpha, \beta, \gamma,]}\right. \\
\left.+\left(1-\delta_{1}^{k}\right)\left(\alpha_{n}^{2}-\alpha_{n} \alpha-\beta_{n}^{2}+\beta_{n} \beta-\gamma_{n}^{2}+\gamma_{n} \gamma\right)\right] \\
\times\left. w^{2\left(h^{[\alpha, \beta, \gamma,]}+n-k+1\right)} B_{k-1}\left(x_{1}, \ldots x_{k}\right)\right|_{x_{j}=(-1)^{j} 2(k-1) ! w^{j} ; j=1, \ldots, k} \\
\times\left\{\sum_{l=1}^{n-k+1} \sum_{m=0}^{n-k+1-l} \frac{(-1)^{m}}{(n-k-l+2) !(l+m) !}\right. \\
\times\left[\left(l-\delta_{1}^{l}\right) \tilde{h}^{\left[\alpha_{n}, \beta_{n}, \gamma_{n}\right]}+\delta_{1}^{l} \tilde{h}^{[\alpha, \beta, \gamma,]}+\left(1-\delta_{1}^{l}\right)\left(\alpha_{n}^{2}-\alpha_{n} \alpha-\beta_{n}^{2}+\beta_{n} \beta-\gamma_{n}^{2}+\gamma, \gamma\right)\right] \\
\times\left[\partial^{l+m} c B_{001 \mid \alpha_{n} \beta_{n} \gamma_{n}}^{n-k-l-m+2 \mid n-k-l+2} e^{[\alpha, \beta, \gamma]}(w)\right\} \\
+\sum_{k=1}^{n} \frac{(-1)^{k}}{(k-1) !}\left[\frac{\partial^{k+1} c}{k+1} B_{001 \mid \alpha_{n} \beta_{n} \gamma_{n}}^{n-k \mid n} e^{[\alpha, \beta, \gamma]}(w)\right. \\
+\frac{\partial^{k} c}{k} \partial\left(B_{001 \mid \alpha_{n} \beta_{n} \gamma_{n}}^{n-k \mid n} e^{[\alpha, \beta, \gamma]}(w)\right)+c \partial\left(B_{n}^{\left[\alpha_{n}, \beta_{n}, \gamma_{n}\right]} e^{[\alpha, \beta, \gamma]}\right)(w)
\end{array}
$$

Here $B_{p q r \mid \alpha \beta \gamma}^{m \mid n}$ are the conformal dimension $m$ polynomials in bosonized ghost fields appearing in the OPE of Bell polynomials with exponential fields, defined according to:

$$
B_{n}^{\left[\alpha_{n}, \beta_{n}, \gamma_{n},\right]}\left(z_{1}\right) e^{[p, q, r]}\left(z_{2}\right)=\sum_{m=0}^{n} \frac{: B_{p q r \mid \alpha \beta \gamma}^{m \mid n}\left(z_{1}\right) e^{[p, q, r]}\left(z_{2}\right):}{\left(z_{1}-z_{2}\right)^{n-m}}
$$

(note the upper script for $B^{m \mid n}$ chosen here in order not to confuse them with the incomplete Bell polynomials for which the lower indices are reserved according to (15)) It is straightforward to compute the manifest expressions for $B_{p q r \mid \alpha \beta \gamma}^{m \mid n}$ in terms of incomplete Bell polynomials. Using the representation (15) for the Bell polynomials in terms of partitions, computing the OPE (42) and extracting the relevant coefficients we get

$$
B_{p q r \mid \alpha \beta \gamma}^{m \mid n}=\frac{(-1)^{n-m} n !}{(n-m) ! m !} \sum_{k=1}^{n} \sum_{l=\max (1 ; k-m)}^{\min (n-m ; k)} B_{n-m \mid l}(0 !, 1 !, \ldots,(n-m-l) !) B_{m \mid k-l}^{[\alpha, \beta, \gamma]}
$$


Here $B_{m \mid k-l}^{[\alpha, \beta, \gamma]}$ are the incomplete Bell polynomials in the ghost fields defined according to (10), (14). The numerical coefficients $B_{n-m \mid l}(0 !, 1 !, \ldots,(n-m-l) !)$ given by the values of incomplete Bell polynomials $B_{n-m \mid l}\left(x_{1}, \ldots x_{n-m-l+1}\right)$ at $x_{j}=(j-1) !(j=1, \ldots ; n-m-l+1)$ and coincide with $(n-m)$ 'th order expansion coefficients of $\log ^{l}(1+x)$ around $x=0$.

\section{Computation of the Star Product}

Finally, before starting the computation of the correlators, we shall comment on cohomology constraints on $\Psi^{(+)}$and $\Psi^{(-)}$, playing the role of fixing the gauge in SFT and defining the limits of summation over $n$ in (9). Since the analytic solution we are looking for, is aiming to describe the higher spin vacuum, and the higher spin algebras are determined by the structure of OPE's between ghost cohomologies $H_{n} \sim H_{-n-2}(n>0)$, we impose the following weak cohomology constraints according to:

$$
\begin{array}{r}
\Gamma(z) \Psi^{(-)}(w) \sim O(z-w)^{0} \\
\Gamma(z) \Psi^{(-)} \approx 0
\end{array}
$$

The first of these conditions ensures that $\Psi^{(-)}$is nonsingular under the picture-changing transformation; the second constraint requires that $\Psi^{(-)}$is annihilated by $\Gamma$ in a weak sense, that is, up to terms not contributing to the correlation functions we are considering. Technically, this implies that, with the picture-changing operator:

$$
\Gamma=-\frac{1}{2} e^{\phi} \psi_{m} \partial X^{m}+\frac{1}{4} b e^{2 \phi-\chi}(\partial \chi+\partial \sigma)+c e^{\chi} \partial \chi
$$

$\Psi^{(-)}$is annihilated by the first two terms of $\Gamma$ but is allowed to have a nonvanishing nonsingular OPE with the last one. However, as the transformation by $c \partial \xi$ shifts the $b-c$ and $\xi-\eta$ ghost numbers of the string field components by 1 unit, the terms obtained as a result of the picture-changing will not contribute to the correlators. Since positive and negative cohomologies are isomorphic, it is sufficient to consider the constraints on $\Psi^{(-)}$ The constraints (44) ensure that string field components components of different ghost numbers are unrelated by picture changing (up to terms irrelevant for correlators). This leads to

$$
\begin{array}{r}
\beta_{n}=0 \\
\gamma_{n}=0 ; 1 \\
n \leq N-1
\end{array}
$$

With the constraints $(44) \Psi^{(+)}$is also automatically annihilated by the inverse picture changing operator $\Gamma^{-1}$, at least in the weak sense. This condition is stronger than standard 
gauge constraints on string fields. Note that the vanishing of $\beta_{n}$ particularly ensures the standard gauge condition $\eta_{0} \Psi=0$. This is the condition typically imposed on string fields at particular fixed ghost number. In our case, however, the constraints clearly have to be stronger than that since we allowed the contributions from all the ghost numbers. Note that the condition $\beta_{n}=0$ also technically reduces the string field (9) to the small Hilbert space (although for generic $\alpha_{n}, \beta_{n}, \gamma_{n} \Psi$ belongs to the large space). Indeed, the only

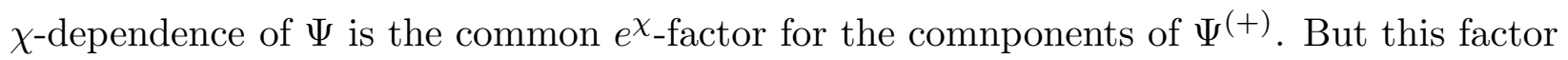
simply ensures the cancellation of the $\chi$-ghost's background charge and does not affect the rest of the calculations which are effectively in the small space. Also, in our calculation of the correlators we fix the gauge $\alpha_{n}=n$. This is done for simplicity of our calculations; it is straightforward to generalize them for arbitrary $\alpha_{n}$. Finally, we find that the $\gamma_{n}=1$ choice in (46) is the only one leading to nontrivial recursion relation on $\lambda_{N}^{n}$-coefficients in (11) with the opposite the $\gamma_{n}=0$ choice trivializing the correlators and destroying the general structure of the solutions. With $I \circ Q \Psi$ determined, we are now prepared to calculate $<<\Psi, Q \Psi>>$ for the string field (9).

To compute the correlators, the following operator products are of importance:

$$
\begin{gathered}
B_{n}^{[\alpha, \beta, \gamma]}(z) e^{[p, q, r]}(w)=\sum_{k=1}^{n} \sum_{l=o}^{k} \sum_{m=l}^{n-k+l}(z-w)^{-m} \\
\times \frac{n !}{m !(n-m) !} B_{m \mid l}(0 !, 1 !, \ldots(m-l) !): B_{n-m \mid k-l}^{[\alpha, \beta, \gamma]}(z) e^{[p, q, r]}(w): \\
B_{n_{1} \mid k_{1}}^{[\alpha, \beta, \gamma]}(z) B_{n_{2} \mid k_{2}}^{[p, q, r]}(w)=\sum_{l=0}^{m i n\left(k_{1}, k_{2}\right)} \sum_{m_{1}=l}^{n_{1}-k_{1}+l} \sum_{m_{2}=l}^{n_{2}-k_{2}+l}(z-w)^{-m_{1}-m_{2}} \frac{n_{1} ! n_{2} !}{\left(n_{1}-m_{1}\right) !\left(n_{2}-m_{2}\right) !} \\
\Lambda_{B e l l}\left(m_{1}, m_{2} \mid l\right): B_{n_{1}-m_{1} \mid k_{1}-l}^{[\alpha, \beta, \gamma]}(z) B_{n_{2}-m_{2} \mid k_{2}-l}^{[p, q, r]}(w)
\end{gathered}
$$

where the signs of the normal ordering imply the absence of contractions on the right hand side and the generalized Bell numbers

$\Lambda_{\text {Bell }}\left(m_{1}, m_{2} \mid l\right)$ are defined as follows. Let $0<p_{1} \leq p_{2} \ldots \leq p_{l}$ and $0<q_{1} \leq p_{2} \ldots \leq q_{l}$

be the ordered length $l$ partitions of $m_{1}$ and $m_{2}$. Then

$$
\begin{gathered}
\Lambda_{\text {Bell }}\left(m_{1}, m_{2} \mid l\right)=m_{1} ! m_{2} ! \sum_{m_{1} \mid p_{1}, \ldots, p_{l}}^{\text {partitions }} \sum_{m_{2} \mid q_{1}, \ldots, q_{l}}^{\text {partitions }} \\
\sum_{p_{i_{k} ; q_{j_{k}} ; i_{k}, j_{k}=1, \ldots, l}}^{p_{1} \ldots p_{1} p_{l} ! q_{1} ! \ldots q_{l} ! r_{p_{1}} ! \ldots r_{p_{l}} ! r_{q_{1}} ! \ldots r_{q_{l}} !}
\end{gathered}
$$


where

$r_{p, q}$ are multiplicities of $p$ and $q$ entering the partitions.

The ghost number anomaly cancellation condition requires that each correlator (both 2 -point and 3-point) must have $b-c$ ghost number $3, \phi$-ghost number -2 and $\chi$-ghost number 1 . It is this condition that ensures the triangular form of (11), making it a well-defined recurrence relation.

The straightforward calculation of $<<\Psi, Q \Psi>>$ gives

$$
\begin{aligned}
& <<\Psi, Q \Psi>> \\
& =\sum_{N=1}^{\infty} \sum_{n=0}^{N-1}\left(\lambda_{N}^{n}\right)^{2} \sum_{k=1}^{n+1} \sum_{l=1}^{n-k+1} \sum_{m=0}^{n-k-l} \sum_{L_{1}=0}^{n} \frac{(n !)^{2}}{(n-k-l-m+2) !(l+m-1) !\left(n-L_{1}\right) !} \\
& \left\{\left[\left(k-\delta_{1}^{k}\right) h^{[n, 0,0]}+\delta_{1}^{k} h^{[-(N+2), 0,1]}+\left(1-\delta_{1}^{k}\right)((N+2)(n+1)-1)\right]\right. \\
& \times\left[\left(l-\delta_{1}^{l}\right) \tilde{h}^{[n, 0,0]}+\delta_{1}^{l} \tilde{h}^{[-(N+2), 0,1]}+\left(1-\delta_{1}^{l}\right)((N+2)(n+1)-1)\right] \\
& \times \sum_{k_{1}=1}^{n} \sum_{k_{2}=1}^{l+m-1} \sum_{k_{3}=1}^{n-k-l+2} \sum_{l_{1}=1}^{\min \left(L_{1} ; k_{1}-1\right)} \sum_{l_{2}=1}^{\min \left(m, k_{3}-1\right)}(-1)^{k+l_{1}+l_{2}+L_{1}} \\
& (2+n(N+2))^{l_{1}} B_{L_{1} \mid l_{1}}\left(0 !, \ldots,\left(L_{1}-l_{1}\right) !\right) B_{L_{2} \mid l_{2}}\left(0 !, \ldots,\left(L_{2}-l_{2}\right) !\right) \\
& \times \sum_{q=1}^{k_{3}-l_{3}} \sum_{M=1}^{n+2-k-l-m-k_{3}+l_{2}-q}(n N-1)^{q} B_{M \mid q}(0 !, \ldots,(M-q) !) \\
& \times \sum_{Q=k_{3}-l_{2}-q}^{n-L_{1}-k_{1}-l_{1}-k_{3}+l_{2}+q} \frac{(-1)^{Q+n-L_{1}}\left(n-L_{1}\right) !}{Q !\left(n-L_{1}-Q\right) !} \Lambda_{B e l l}\left(Q ; n+2-k-l-m-M \mid k_{3}-l_{3}-q\right) \\
& \times\left[n^{k_{2}} \delta_{k_{2}}^{k_{1}-k_{3}-l_{1}+l_{2}+q} \Lambda_{\text {Bell }}\left(n-L_{1}-Q ; l+m-1 \mid k_{2}\right)\right. \\
& \left.\left.-(l+m-1) n^{k_{2}-1} \delta_{k_{2}-1}^{k_{1}-k_{3}-l_{1}+l_{2}+q} \Lambda_{B e l l}\left(n-L_{1}-Q ; l+m-2 \mid k_{2}\right)\right]\right\}
\end{aligned}
$$

The next step is to compute the 3-point correlator

$$
<<\Psi, \Psi \star \Psi=<h \circ f_{1}^{3} \circ \Psi(0) h \circ f_{2}^{3} \circ \Psi(0) h \circ f_{3}^{3} \circ \Psi(0)>
$$

where, for the convenience of the computation the conformal transformation

$$
h(z)=-i \frac{z-1}{z+1}
$$

further maps the disc to the half-plane (upon mapping the worldsheets of 3 interacting strings to the disc). The straightforward computation of the 3-point function, using the 
operator products $(47),(48)$ gives

$<<\Psi, \Psi \star \Psi>>=$

$$
\begin{array}{r}
\sum_{N=1}^{\infty} \sum_{N_{1}, N_{2}=1}^{N_{1}+N_{2}=N-2} \lambda_{N}^{n} \lambda_{N_{1}}^{n_{1}} \lambda_{N_{2}}^{n_{2}} \sum_{k=1}^{n+1} \sum_{k_{1}=1}^{n_{1}+1} \sum_{k_{2}=1}^{n_{2}+1} \frac{n ! n_{1} ! n_{2} !}{(n-k+1) !\left(n_{1}-k_{1}+1\right) !\left(n_{2}-k_{2}+1\right) ! k ! k_{1} ! k_{2} !} \\
\times\left[\left(k-\delta_{1}^{k}\right) h^{[n, 0,1]}+\delta_{1}^{k} h^{[N, 0,1]}+\left(1-\delta_{1}^{k}\right)\left(n^{2}-n N\right)\right] \\
\times\left[\left(k_{1}-\delta_{1}^{k_{1}}\right) h^{\left[n_{1}, 0,1\right]}+\delta_{1}^{k} h^{\left[-N_{1}-2,0,1\right]}+\left(1-\delta_{1}^{k_{1}}\right)\left(n_{1}^{2}+n_{1}\left(N_{1}+2\right)\right)\right] \\
\times\left[\left(k_{2}-\delta_{1}^{k_{2}}\right) h^{\left[n_{2}, 0,1\right]}+\delta_{1}^{k_{2}} h^{\left[-N_{2}-2,0,1\right]}+\left(1-\delta_{1}^{k_{2}}\right)\left(n_{2}^{2}+n_{2}\left(N_{2}+2\right)\right)\right] \\
\left(\frac{2}{3}\right)^{h^{[N, 1,1]}+h^{\left[-N_{1}-2,0,1\right]}+h^{\left[-N_{2}-2,0,1\right]}+n+n_{1}+n_{2}-k-k_{1}-k_{2}} \\
\times\left. B_{k-1}\left(\lambda\left(k, n, h^{[n, 0,1]}\right) \log \left(h \circ\left(f_{1}^{3}\right)^{\prime}(z)\right)\right)\right|_{z=0}
\end{array}
$$

$\left.\left.B_{k_{1}-1}\left(\lambda\left(k_{1}, n_{1}, h^{\left[n_{1}, 0,1\right]}\right) \log \left(h \circ\left(f_{2}^{3}\right)^{\prime}(z)\right)\right)\right|_{z=0} B_{k_{2}-1}\left(\lambda\left(k_{2}, n_{2}, h^{\left[n_{2}, 0,1\right]}\right) \log \left(h \circ\left(f_{3}^{3}\right)^{\prime}(z)\right)\right)\right|_{z=0}$

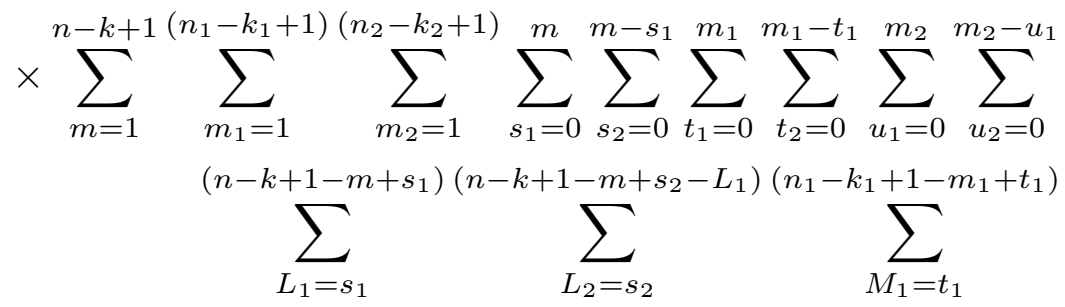

$\left(n_{1}-k_{1}+1-m_{1}+t_{2}-M_{1}\right)\left(n_{2}-k_{2}+1-m_{2}+u_{1}\right)\left(n_{2}-k_{2}+1-m_{2}+u_{2}-P_{1}\right)$

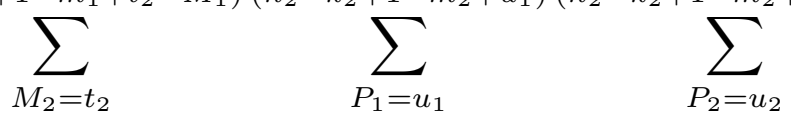

$\left\{B_{L_{1} \mid s_{1}}\left(0 !, 1 !, \ldots,\left(L_{1}-s_{1}\right) !\right) B_{L_{2} \mid s_{2}}\left(0 !, 1 !, \ldots,\left(L_{2}-s_{2}\right) !\right) B_{M_{1} \mid t_{1}}\left(0 !, 1 !, \ldots,\left(M_{1}-t_{1}\right) !\right)\right.$

$B_{M_{2} \mid t_{2}}\left(0 !, 1 !, \ldots,\left(M_{2}-t_{2}\right) !\right) B_{P_{1} \mid u_{1}}\left(0 !, 1 !, \ldots,\left(P_{1}-u_{1}\right) !\right) B_{P_{2} \mid u_{2}}\left(0 !, 1 !, \ldots,\left(P_{2}-u_{2}\right) !\right)$

$$
\begin{array}{r}
\left(-\left(N_{1}+2\right) n-1\right)^{L_{1}}\left(-\left(N_{2}+2\right) n-1\right)^{L_{2}}\left(-n_{1} N+1\right)^{M_{1}} \\
\left(-n_{1}\left(N_{2}+2\right)-1\right)^{M_{2}}\left(-n_{2} N-1\right)^{P_{1}}\left(n_{2}\left(N_{1}+2\right)+1\right)^{P_{2}} \\
\times\left(-n n_{1}+1\right)^{r_{1}}\left(-n n_{2}+1\right)^{r_{2}}\left(-n_{1} n_{2}+1\right)^{r_{3}} \sum_{R_{1}=r_{1}}^{n-k+1+r_{1}} \sum_{R_{2}=r_{2}}^{n_{1}-k_{1}+1+r_{2}} \sum_{R_{3}=r_{3}}^{n_{2}-k_{2}+1+r_{3}} \\
\left.(\sqrt{3})^{-R_{1}+R_{3}}(2 \sqrt{3})^{-R_{1}-R_{3}} \Lambda_{\text {Bell }}\left(R_{1} ; R_{2} \mid r_{1}\right) \Lambda_{\text {Bell }}\left(R_{1} ; R_{3} \mid r_{2}\right) \Lambda_{\text {Bell }}\left(R_{2} ; R_{3} \mid r_{3}\right)\right\}
\end{array}
$$

where 


$$
\begin{array}{r}
r_{1}=\frac{1}{2}\left(m+m_{1}-m_{2}-s_{1}-s_{2}-t_{1}-t_{2}+u_{1}+u_{2}\right) \\
r_{2}=\frac{1}{2}\left(m-m_{1}+m_{2}-s_{1}-s_{2}+t_{1}+t_{2}-u_{1}-u_{2}\right) \\
r_{1}=\frac{1}{2}\left(-m+m_{1}-m_{2}+s_{1}+s_{2}-t_{1}-t_{2}+u_{1}+u_{2}\right)
\end{array}
$$

The values of the complete Bell polynomials appearing as a result of conformal transformations by $h \circ f_{j}^{3}(j=1,2,3)$ in the cubic term (52), are calculated to be given by

$$
\begin{aligned}
B_{k}(\lambda(k, n, & \left.\left.h^{[n, 0,1]}\right) \log \left(h \circ\left(f_{j}^{3}\right)^{\prime}(z)\right)\right)\left.\right|_{z=0}=\sum_{p=0}^{k} \sum_{l=0}^{p} \sum_{m=0}^{k-p} \frac{i^{p+1}(-1)^{l+m} k !}{l !(p-l) ! m !(k-p-m) !} \\
& \times \frac{\Gamma\left(\lambda-\frac{2}{3}\right) \Gamma\left(\lambda+\frac{2}{3}\right)(\Gamma(\lambda+1))^{2}}{\Gamma\left(\lambda-\frac{2}{3}-l\right) \Gamma\left(\lambda+\frac{2}{3}+l-k\right) \Gamma(\lambda+1-m) \Gamma(\lambda+1+m+p-k)}
\end{aligned}
$$

Combining our results for two-point and three-point correlators (50)-(52) we deduce the following recurrence relation for the $\lambda_{N}^{n}$ structure constants entering the analytic solutions:

$$
\rho_{N \mid L N_{1} ; N_{2}}^{n \mid n_{1} ; n_{2}}=\frac{\left(\kappa_{3}\right)_{N \mid N_{1} ; N_{2}}^{n \mid n_{1} ; n_{2}}}{\left(\kappa_{2}\right)_{N}^{n}}
$$

with

$$
\begin{aligned}
& \left(\kappa_{2}\right)_{N}^{n}=\sum_{k=1}^{n+1} \sum_{l=1}^{n-k+1} \sum_{m=0}^{n-k-l} \sum_{L_{1}=0}^{n} \frac{(n !)^{2}}{(n-k-l-m+2) !(l+m-1) !\left(n-L_{1}\right) !} \\
& \left\{\left[\left(k-\delta_{1}^{k}\right) h^{[n, 0,0]}+\delta_{1}^{k} h^{[-(N+2), 0,1]}+\left(1-\delta_{1}^{k}\right)((N+2)(n+1)-1)\right]\right. \\
& \times\left[\left(l-\delta_{1}^{l}\right) \tilde{h}^{[n, 0,0]}+\delta_{1}^{l} \tilde{h}^{[-(N+2), 0,1]}+\left(1-\delta_{1}^{l}\right)((N+2)(n+1)-1)\right] \\
& \times \sum_{k_{1}=1}^{n} \sum_{k_{2}=1}^{l+m-1} \sum_{k_{3}=1}^{n-k-l+2} \sum_{l_{1}=1}^{\min \left(L_{1} ; k_{1}-1\right)} \sum_{l_{2}=1}^{\min \left(m, k_{3}-1\right)}(-1)^{k+l_{1}+l_{2}+L_{1}} \\
& (2+n(N+2))^{l_{1}} B_{L_{1} \mid l_{1}}\left(0 !, \ldots,\left(L_{1}-l_{1}\right) !\right) B_{L_{2} \mid l_{2}}\left(0 !, \ldots,\left(L_{2}-l_{2}\right) !\right) \\
& \times \sum_{q=1}^{k_{3}-l_{3}} \sum_{M=1}^{n+2-k-l-m-k_{3}+l_{2}-q}(n N-1)^{q} B_{M \mid q}(0 !, \ldots,(M-q) !) \\
& \times \sum_{Q=k_{3}-l_{2}-q}^{n-L_{1}-k_{1}-l_{1}-k_{3}+l_{2}+q} \frac{(-1)^{Q+n-L_{1}}\left(n-L_{1}\right) !}{Q !\left(n-L_{1}-Q\right) !} \Lambda_{B e l l}\left(Q ; n+2-k-l-m-M \mid k_{3}-l_{3}-q\right) \\
& \times\left[n^{k_{2}} \delta_{k_{2}}^{k_{1}-k_{3}-l_{1}+l_{2}+q} \Lambda_{\text {Bell }}\left(n-L_{1}-Q ; l+m-1 \mid k_{2}\right)\right. \\
& \left.\left.-(l+m-1) n^{k_{2}-1} \delta_{k_{2}-1}^{k_{1}-k_{3}-l_{1}+l_{2}+q} \Lambda_{B e l l}\left(n-L_{1}-Q ; l+m-2 \mid k_{2}\right)\right]\right\}
\end{aligned}
$$


and

$$
\begin{aligned}
& \left(\kappa_{3}\right)_{N \mid N_{1} ; N_{2}}^{n \mid n_{1} ; n_{2}}=\sum_{k=1}^{n+1} \sum_{k_{1}=1}^{n_{1}+1} \sum_{k_{2}=1}^{n_{2}+1} \frac{n ! n_{1} ! n_{2} !}{(n-k+1) !\left(n_{1}-k_{1}+1\right) !\left(n_{2}-k_{2}+1\right) ! k ! k_{1} ! k_{2} !} \\
& \times\left[\left(k-\delta_{1}^{k}\right) h^{[n, 0,1]}+\delta_{1}^{k} h^{[N, 0,1]}+\left(1-\delta_{1}^{k}\right)\left(n^{2}-n N\right)\right] \\
& \times\left[\left(k_{1}-\delta_{1}^{k_{1}}\right) h^{\left[n_{1}, 0,1\right]}+\delta_{1}^{k} h^{\left[-N_{1}-2,0,1\right]}+\left(1-\delta_{1}^{k_{1}}\right)\left(n_{1}^{2}+n_{1}\left(N_{1}+2\right)\right)\right] \\
& \times\left[\left(k_{2}-\delta_{1}^{k_{2}}\right) h^{\left[n_{2}, 0,1\right]}+\delta_{1}^{k_{2}} h^{\left[-N_{2}-2,0,1\right]}+\left(1-\delta_{1}^{k_{2}}\right)\left(n_{2}^{2}+n_{2}\left(N_{2}+2\right)\right)\right] \\
& \left(\frac{2}{3}\right)^{h^{[N, 1,1]}+h^{\left[-N_{1}-2,0,1\right]}+h^{\left[-N_{2}-2,0,1\right]}+n+n_{1}+n_{2}-k-k_{1}-k_{2}} \\
& \times\left. B_{k-1}\left(\lambda\left(k, n, h^{[n, 0,1]}\right) \log \left(h \circ\left(f_{1}^{3}\right)^{\prime}(z)\right)\right)\right|_{z=0} \\
& \left.\left.B_{k_{1}-1}\left(\lambda\left(k_{1}, n_{1}, h^{\left[n_{1}, 0,1\right]}\right) \log \left(h \circ\left(f_{2}^{3}\right)^{\prime}(z)\right)\right)\right|_{z=0} B_{k_{2}-1}\left(\lambda\left(k_{2}, n_{2}, h^{\left[n_{2}, 0,1\right]}\right) \log \left(h \circ\left(f_{3}^{3}\right)^{\prime}(z)\right)\right)\right|_{z=0} \\
& \times \sum_{m=1}^{n-k+1} \sum_{m_{1}=1}^{\left(n_{1}-k_{1}+1\right)} \sum_{m_{2}=1}^{\left(n_{2}-k_{2}+1\right)} \sum_{s_{1}=0}^{m} \sum_{s_{2}=0}^{m-s_{1}} \sum_{t_{1}=0}^{m_{1}} \sum_{t_{2}=0}^{m_{1}-t_{1}} \sum_{u_{1}=0}^{m_{2}} \sum_{u_{2}=0}^{m_{2}-u_{1}} \\
& \left(n-k+1-m+s_{1}\right)\left(n-k+1-m+s_{2}-L_{1}\right)\left(n_{1}-k_{1}+1-m_{1}+t_{1}\right) \\
& \sum_{L_{1}=s_{1}} \sum_{L_{2}=s_{2}} \sum_{M_{1}=t_{1}} \\
& \left(n_{1}-k_{1}+1-m_{1}+t_{2}-M_{1}\right)\left(n_{2}-k_{2}+1-m_{2}+u_{1}\right)\left(n_{2}-k_{2}+1-m_{2}+u_{2}-P_{1}\right) \\
& \sum_{M_{2}=t_{2}} \sum_{P_{1}=u_{1}} \sum_{P_{2}=u_{2}} \\
& \left\{B_{L_{1} \mid s_{1}}\left(0 !, 1 !, \ldots,\left(L_{1}-s_{1}\right) !\right) B_{L_{2} \mid s_{2}}\left(0 !, 1 !, \ldots,\left(L_{2}-s_{2}\right) !\right) B_{M_{1} \mid t_{1}}\left(0 !, 1 !, \ldots,\left(M_{1}-t_{1}\right) !\right)\right. \\
& B_{M_{2} \mid t_{2}}\left(0 !, 1 !, \ldots,\left(M_{2}-t_{2}\right) !\right) B_{P_{1} \mid u_{1}}\left(0 !, 1 !, \ldots,\left(P_{1}-u_{1}\right) !\right) B_{P_{2} \mid u_{2}}\left(0 !, 1 !, \ldots,\left(P_{2}-u_{2}\right) !\right) \\
& \left(-\left(N_{1}+2\right) n-1\right)^{L_{1}}\left(-\left(N_{2}+2\right) n-1\right)^{L_{2}}\left(-n_{1} N+1\right)^{M_{1}} \\
& \left(-n_{1}\left(N_{2}+2\right)-1\right)^{M_{2}}\left(-n_{2} N-1\right)^{P_{1}}\left(n_{2}\left(N_{1}+2\right)+1\right)^{P_{2}} \\
& \left.(\sqrt{3})^{N\left(N_{1}+2\right)-\left(N_{1}+2\right)\left(N_{2}+1\right)+2-L_{1}-M_{1}-M_{2}-P_{2}}(2 \sqrt{3})^{N\left(N_{2}+2\right)+1-L_{2}-P_{1}}\right\} \\
& \times\left(-n n_{1}+1\right)^{r_{1}}\left(-n n_{2}+1\right)^{r_{2}}\left(-n_{1} n_{2}+1\right)^{r_{3}} \sum_{R_{1}=r_{1}}^{n-k+1+r_{1}} \sum_{R_{2}=r_{2}}^{n_{1}-k_{1}+1+r_{2}} \sum_{R_{3}=r_{3}}^{n_{2}-k_{2}+1+r_{3}} \\
& \left.(\sqrt{3})^{-R_{1}+R_{3}}(2 \sqrt{3})^{-R_{1}-R_{3}} \Lambda_{\text {Bell }}\left(R_{1} ; R_{2} \mid r_{1}\right) \Lambda_{\text {Bell }}\left(R_{1} ; R_{3} \mid r_{2}\right) \Lambda_{\text {Bell }}\left(R_{2} ; R_{3} \mid r_{3}\right)\right\}
\end{aligned}
$$

This concludes the computation of the coefficients defining the analytic OSFT solution.

\section{Bell Polynomials and Higher Spin Algebras}

In this concluding section we shall present some arguments relating the structure of the analytic OSFT solution, studied in this work, to free field realizations of the higher spin algebras in $A d S$. An insightful hint, relating Bell polynomials to free field realizations 
of higher spin algebras in AdS, comes from $c=1$ model, i.e. one-dimensional noncritical string theory.

The one-dimensional string compactified on $S^{1}$ has no standard massless modes (like a photon) but does have a $S U(2)$ multiplet of massless states existing at nonstandard $b-c$ ghost numbers and discrete mumentum values [35], [36], [12]

The $S U(2)$ symmetry at self-dual radius $R=\frac{1}{\sqrt{2}}$ is realized by the operators:

$$
T_{ \pm}=\oint d z e^{ \pm i X \sqrt{2}} ; T_{0}=\oint d z \partial X
$$

The SU(2) multiplet of discrete states can be constructed by acting with the lowering $T_{-}$of $S U(2)$ on the highest weight vectors given by tachyonic primaries

$$
V_{l}=e^{(i l X+(l-1) \varphi) \sqrt{2}}:(\text { with integer } 1)
$$

$$
U_{l \mid m}=T_{-}^{l-m} V_{l}
$$

Manifest expressions for $U_{l \mid m}$ vertex operators are complicated, however, their structure constants have been deduced by [35], [36] by using symmetry arguments. One has

$$
U_{l_{1} \mid m_{1}}(z) U_{l_{2} \mid m_{2}}(w) \sim(z-w)^{-1} C\left(l_{1}, l_{2}, l_{3} \mid m_{1}, m_{2}, m_{3}\right) f\left(l_{1}, l_{2}\right) U_{l_{3}, m_{3}}
$$

where the $S U(2)$ Clebsch-Gordan coefficients are fixed by the symmetry while the function of Casimir eigenvalues $f\left(l_{1}, l_{2}\right)$ is nontrivial and was deduced to be given by

$$
f\left(l_{1}, l_{2}\right)=\frac{\sqrt{l_{1}+l_{2}}\left(2 l_{1}+2 l_{2}-2\right) !}{\sqrt{2 l_{1} l_{2}}\left(2 l_{1}-1\right) !\left(2 l_{2}-1\right) !}
$$

35, 36

Remarkably, these structure constants coincide exactly with those of higher spin algebra in $A d S_{3}$ in a certain basis, computed by E. Fradkin and V. Linetsky in 1989, in a seemingly different context [37], [38] On the other hand, the explicit structure of the vertex operators for the discrete states realizing this algebra is given by

$$
U_{l \mid m} \sim \sum_{\frac{1}{2}(l(l-1)-m(m-1)) \mid p_{1}, \ldots, p_{l-m}} B_{p_{1}}(-i X \sqrt{2}) \ldots B_{p_{l-m}}(-i X \sqrt{2}) e^{\sqrt{2}(i m X+(l-1) \varphi)}
$$

with the sum taken over ordered partitions of $\frac{1}{2}(l(l-1)-m(m-1)) \mid p_{1}, \ldots, p_{l-m}$ 
This is a relatively simple example of Bell polynomials multiplied by exponentials realizing the higher spin algebras in $A d S_{d}$ in terms of vertex operator algebras in $d-1$ dimensional string theory. One can further think of the extending the symmetry of the $c=1$ model by supersymmetrizing it on the worldsheet and coupling to the $\beta-\gamma$ enhancing the symmetry from $S U(2)$ to $S U(4)$ The $\mathrm{SU}(4)$ algebra can be realized by taking the raising generators:

$$
\begin{aligned}
T 0 ; 1=\oint d z e^{i X} \psi T^{-3 ; 2} & =\oint d z e^{-3 \phi+2 i X} \psi \\
T^{-4 ; 3} & =\oint d z e^{-4 \phi+3 i X} \psi
\end{aligned}
$$

and the remaining 12 generators are obtained by acting on (63) with the lowering generators of $S U(2), T^{0 ;-1}=\oint d z e^{-i X} \psi$ :

$$
\begin{array}{r}
T^{-4 ; k}=\left(T^{0 ;-1}\right)^{3-k} T^{-4 ; 3} \\
T^{-3 ; l}=\left(T^{0 ;-1}\right)^{2-k} T^{-3 ; 2} \\
T^{0 ; m}=\left(T^{0 ;-1}\right)^{1-k} T^{0 ; 1} \\
-3 \leq k \leq 3 \\
-2 \leq l \leq 2 \\
-1 \leq m \leq 1
\end{array}
$$

with $T^{0 ; 0}, T^{-3 ; 0} \cdot T^{-4 ; 0}$ being the Cartan generators of $S U(4)$. The $S U(4)$ multiplet is then obtained by the combinations of lowering operators of $S U(4)$ with negative $k, l, m$ acting on the same dressed tachyonic primaries entering (59). The structure constants of the operators of the $S U(4)$ are again given by the Klebsch-Gordan coefficients of $S U(4)$ multiplied by certain functions of the eigenvalues of SU(4) Casimir operators. Unfortunately, because of the complexity of the $\mathrm{SU}(4)$ operators, the explicit form of these functions has never been worked out. Evaluating them would be emportant in order to point out the relation of this operator algebra to the structure constants of the higher spin algebra in $A d S_{5}$. With the techniques, explained in this paper, we hope to be able to perform this computation in the future work. As in the $S U(2)$ case, the structure of the manifest vertex operator expressions for the $S U(4)$ multiplet involve products of Bell polynomials in $X, \psi$ and ghost fields multiplies by exponents of $X$, although the structure of the Bell polynomials in the $S U(4)$ case is clearly more complicated than (62). Thus one can think of Bell polynomial products (multiplied by exponents) as of natural vertex operator realizations of 
various $A d S$ higher spin algebras in string theory. The string field theory analytic solution presented in this paper is a simple example of this class of the operators. By itself, it is clearly incomplete to describe the full higher spin vacuum, despite some of its attractive properties. In particular, one clearly has to generalize this string field theory solution to include products of multiple Bell polynomials, in order to make connections to the full higher spin algebras in $A d S$. Our particular conjecture is that the OSFT solutions of the type:

$$
\Psi=\sum_{N, n_{1}, \ldots, n_{k}} \lambda_{N}^{n_{1} \ldots n_{k}} B_{n_{1}}(\phi, \chi, \sigma) \ldots B_{n_{k}}(\phi, \chi, \sigma)\left(c \xi e^{N \phi}+c e^{-(N+2) \phi}\right)
$$

can be related to contributions of the $k$-row higher spin fields with mixed symmetries to the collective higher spin vacuum configuration. In general, the full space of these solutions would form an "enveloping" of higher-spin algebra. It would be interesting to point out the connection of this enveloping to multiparticle extensions of the higher spin algebras proposed by Vasiliev [39]. With the on-shell arguments, showing the relevance of the vertex operators for the frame-like higher spin fields to the background independence, relating the languages and concepts of higher spin gauge theories and string field theory promises a fascinating ground for the future work.

\section{Acknowledgements}

It is a pleasure to thank Loriano Bonora and other organizers and participants of SFT-2014 conference at SISSA, Trieste for hospitality and productive discussions.

This work was partially supported by the National Research Foundation of Korea(NRF) grant funded by the Korea government(MEST) through the Center for Quantum Spacetime(CQUeST) of Sogang University with grant number 2005-0049409. I also acknowledge the support of the NRF grant number 2012-004581. 


\section{References}

[1] E. Fradkin, M. Vasiliev, Phys. Lett. B189 (1987) 89

[2] E.S. Fradkin and M.A. Vasiliev, Mod. Phys. Lett. A 3 (1988) 2983

[3] V. E. Lopatin and M. A. Vasiliev, Mod. Phys. Lett. A 3 (1988) 257

[4] A. Sagnotti, M. Taronna, arXiv:1006.5242, Nucl.Phys.B842:299-361,2011

[5] A. Sagnotti, arXiv:1112.4285, J.Phys A46 (2013) 214006

[6] D. Polyakov, Phys.Rev.D82:066005,2010

[7] I. Klebanov, A. M. Polyakov, xPhys.Lett.B550 (2002) 213-219

[8] E. Witten, Nucl.Phys. B268 (1986) 253

[9] E. Witten, Phys.Rev. D46 (1992) 5467-5473

[10] C. Preitschopf, C. Thorn and S. Yost, Nucl. Phys. B337 (1990) 363

[11] I. Arefeva, P. Medvedev, A. Zubarev, Nucl.Phys. B341 (1990) 464-498

[12] I. Arefeva, A. Zubarev, Mod.Phys.Lett. A8 (1993) 1469-1476

[13] N. Berkovits, Nucl. Phys. B450 (1995) 90

[14] N. Berkovits, A. Sen, B. Zwiebach, Nucl.Phys. B587 (2000) 147-178

[15] N. Berkovits, JHEP 0004 (2000) 022

[16] L. Rastelli, B. Zwiebach, JHEP 0109 (2001) 038

[17] L. Bonora, C. Maccaferri, P. Prester, JHEP 0401 (2004) 038

[18] L. Bonora, C. Maccaferri, R. Scherer Santos, D. Tolla, Nucl.Phys. B715 (2005) 413-439

[19] L. Bonora, C. Maccaferri, D. Tolla, JHEP 1111 (2011) 107

[20] I. Bars, Phys.Rev. D66 (2002) 066003

[21] I. Bars, I. Kishimoto, Y. Matsuo, Phys.Rev. D67 (2003) 126007

[22] D. Polyakov, Phys.Rev. D89 (2014) 026010

[23] M. A. Vasiliev, Sov. J. Nucl. Phys. 32 (1980) 439, Yad. Fiz. 32 (1980) 855

[24] E.D. Skvortsov, M.A. Vasiliev, Nucl. Phys.B 756 (2006)117

[25] C. Fronsdal, Phys.Rev. D20 (1979) 848-856

[26] M. Schnabl, Adv.Theor.Math.Phys. 10 (2006) 433-501

[27] T. Erler, M. Schnabl,JHEP 0910 (2009) 066

[28] M. Kroyter, Y. Okawa, M. Schnabl, S. Torii, B. Zwiebach, JHEP 1203 (2012) 030

[29] T. Erler, JHEP 0801:013, (2008)

[30] I. Arefeva, P. Medvedev, A. Zubarev, Mod. Phys. Lett. A6, 949 (1991)

[31] M. Kroyter, JHEP 1103 (2011) 081

[32] E. T. Bell, Annals of Mathematics 29 (1/4): 3846

[33] G. E. Andrews, "The Theory of Partitions" Cambridge University Press ISBN 0-52163766-X. (1998)

[34] K. Boyadzhiev, Abstract and Applied Analysis 2009: Article ID 168672

[35] I. Klebanov, A. Polyakov, Mod.Phys.Lett. A6 (1991) 3273-3281

[36] E. Witten, Nucl.Phys. B373 (1992) 187-213 
[37] E. Fradkin, V. Linetsky, Mod.Phys.Lett. A4 (1989) 2635-2647

[38] E. Fradkin, V. Linetsky, Mod.Phys.Lett. A4 (1989) 2649-2665

[39] M. Vasiliev, Class.Quant.Grav. 30 (2013) 104006 